\title{
Pulmonary blastoma in a pregnant woman: a case report and brief review of the literature
}

\author{
Michał P. Budzik ${ }^{1,2^{*}}$, Grzegorz Panek', Małgorzata Bińkowska', Beata Osuch', Ewa Borkowska ${ }^{3}$ and \\ Anna M. Badowska-Kozakiewicz ${ }^{2}$
}

\begin{abstract} serious pregnancy-related complications. ing pregnancy.

\section{Background}

The symptom of haemoptysis usually indicates a serious pathology and can occur with lung cancer, infections, vasculitis or certain cardiovascular conditions. When occurs in pregnancy, may be assumed to be caused by a pulmonary embolism, which remains the most common haemoptysis cause in pregnant women and is associated with maternal obstetric complications and high fetal risk resulting from intrauterine hypoxia and preterm birth. Although rare, lung tumors should not be overlooked in the presence of haemoptysis during pregnancy, especially considering the rare subtypes of cancer occurring in young women without the usual risk factors for lung cancer.
\end{abstract}

Background: Pulmonary blastoma (PB) comprises a rare heterogeneous group of lung tumours typically containing immature epithelial and mesenchymal structures that imitate the embryonic lung tissue and extremely rarely occurs during pregnancy. Although cough and haemoptysis are the most common PB symptoms, they usually indicate other

Case presentation: The article presents the unusual case of a 22-year-old pregnant woman diagnosed with PB dur-

Conclusions: PB is characterized by poor prognosis and patients' outcome relies on a rapid diagnosis. Surgery remains the most common and effective treatment. Due to the extreme rarity, the literature contains only single mentions of PB in pregnancy, thus its impact on the course of pregnancy and the developing fetus remains unknown.

Keywords: Pulmonary blastoma, Histopathology, Case report, Pregnancy, Lung cancer, Haemoptysis

\footnotetext{
*Correspondence: michalbudzik.5@gmail.com

${ }^{1}$ Department of Gynaecologic Oncology and Obstetrics, Centre

of Postgraduate Medical Education, 231 Czerniakowska, 00-416 Warsaw,

Poland

Full list of author information is available at the end of the article
}

\section{Case presentation}

A 22-year-old woman (gravida 1) was referred to our hospital at 37 weeks' gestation with chest pain, cough and haemoptysis. She had the medical history of SARS$\mathrm{CoV}-2$ asymptomatic infection underwent 3 weeks before. Currently, she was with no reported complications related to pregnancy. Her previous medical and family history was unremarkable, and she was a nonsmoker. On physical examination, her breath sounds were diminished in the right lung zone. Vital signs were BP 112/68 mm Hg, heart rate 84 beats/min, respiratory rate 22 breaths/min, oxygen saturation $97 \%$ on room air and body temperature $36.4{ }^{\circ} \mathrm{C}$. Arterial blood gases were within the normal range for pregnancy, while $\mathrm{pCO} 2$ remained slightly lowered compared to non-pregnant conditions (pO2 $91.8 \mathrm{mmHg}$ [reference range 65.0-99.0], pCO2 $34.4 \mathrm{mmHg}$ [35.0-45.0]). Pregnancy is a state of physiological respiratory alkalosis and compensatory metabolic acidosis. The resulting acid-base disturbances are compensated by the kidneys. Low levels of pCO2 in the maternal arterial blood facilitate the uptake of oxygen 
by the placenta. It is therefore an adaptive change that ensures the optimal concentration of oxygen in the fetal circulation [1]. Complete blood count and complete metabolic panel were normal (e.g. WBC $9.4210^{9} / \mathrm{L}$ [4.010.0]; CRP $5.03 \mathrm{mg} / \mathrm{l}$ [0.00-10.00]; procalcitonin $0.02 \mathrm{ng} /$ $\mathrm{ml}[<0.5 \mathrm{ng} / \mathrm{ml}$-low risk of severe sepsis and/or septic shock; $>2.0 \mathrm{ng} / \mathrm{ml}$-high risk of severe sepsis and/or septic shock]. The PCR test for SARS-CoV-2 mRNA, QuantiFERON-Tuberculosis test and sputum culture were negative. At the presentation to the hospital, due to the patient's stable condition and obtained blood test results, pulmonary embolism and an infectious substrate were excluded. The chest X-rays showed the presence of a solid mass $9 \mathrm{~cm}$ in diameter peripherally in the lower lobe of the right lung with a slight displacement of the mediastinum to the left side and moderate pleural effusion (Fig. 1). Due to the increasing haemoptysis and development of a partial respiratory insufficiency (pO2 $57.5 \mathrm{mmHg}$ [65.099.0], pCO2 $39.8 \mathrm{mmHg}$ [35.0-45.0]), an urgent caesarean section was performed at 38 pregnancy week and a son in good condition was born (10 points on the Apgar score). The patient's condition started to deteriorate due to increased bleeding and development of hypercapnic respiratory failure (pO2 $53.1 \mathrm{mmHg}$ [65.0-99.0], pCO2 $48.3 \mathrm{mmHg}$ [35.0-45.0]) so she was transferred to the thoracic surgery department. Contrast-enhanced chest computed tomography (CT) confirmed a $9.5 \times 8.0 \mathrm{~cm}$ mass in the right lower lobe. The boundary of the lesion was clear and smooth, the enhancement was heterogenous, with evidence numerous necrotic foci. The mass

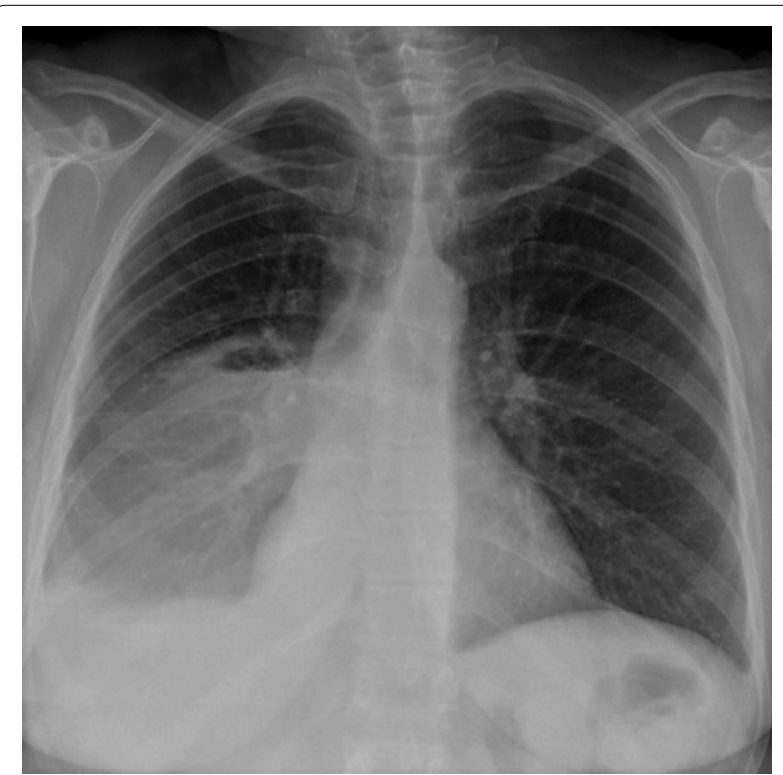

Fig. 1 Chest X-ray showing the presence of solid mass peripherally in the lower lobe of the right lung abutted the pleura with no sign of invasion. There was no evidence of lymphadenopathy or metastases. A slight pleural effusion was described. The tumor was classified as clinical T4NOM0, stage IIIA according to the TNM classification of the Union of International Cancer Control (UICC), 8th edition.

An emergency right-side posterolateral thoracotomy with right lower lobectomy and lymph node dissection was done on the second day after caesarean section.

On gross examination the tumor was well circumscribed unencapsulated mass, measured $10 \times 8 \mathrm{~cm}$. The cut surface of the lesion was heterogeneously white-grey with necrotic and multiple foci of hemorrhagic degeneration. Definitive histology with hematoxylin and eosin (H\&E) staining demonstrated typical biphasic tumor with mixed epithelial and mesenchymal components (Fig. 2). The epithelial component formed glands/tubules with clear cytoplasm, whereas the mesenchymal component consisted of loose undifferentiated mesenchymal stroma with variable cellular atypia and foci of cartilage differentiation. Immunohistochemical (IHC) staining showed CK-5/6 (+) (Fig. 3), CK-7 (+), CD56 and vimentin (stromal cells), desmin $(+)$, CD34 and CD99 (-), synaptophysin (-), myogenin (+) (Fig. 4) and PD-L1 (+) (Fig. 5). High mitotic activity was demonstrated in both epithelial and mesenchymal elements by using Ki-67 (up to 70\%). The surgical margins and lymph nodes were negative.

Postoperative chest radiographs showed optimal lung expansion. Additional imaging studies did not reveal the presence of distant metastases. Subsequent

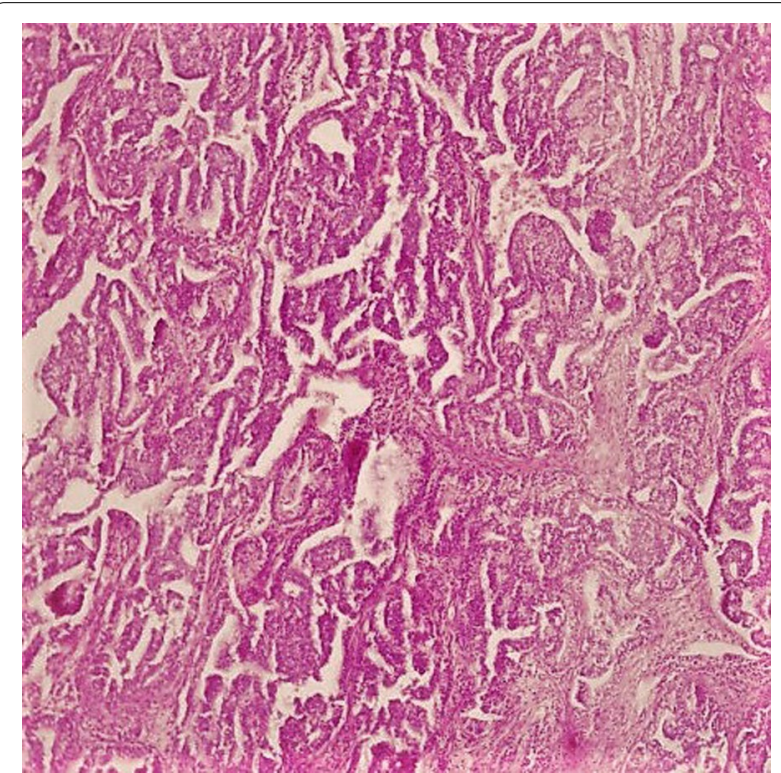

Fig. 2 A biphasic pulmonary blastoma composed of malignant epithelial and mesenchymal component (H\&E, 100× magnification) 


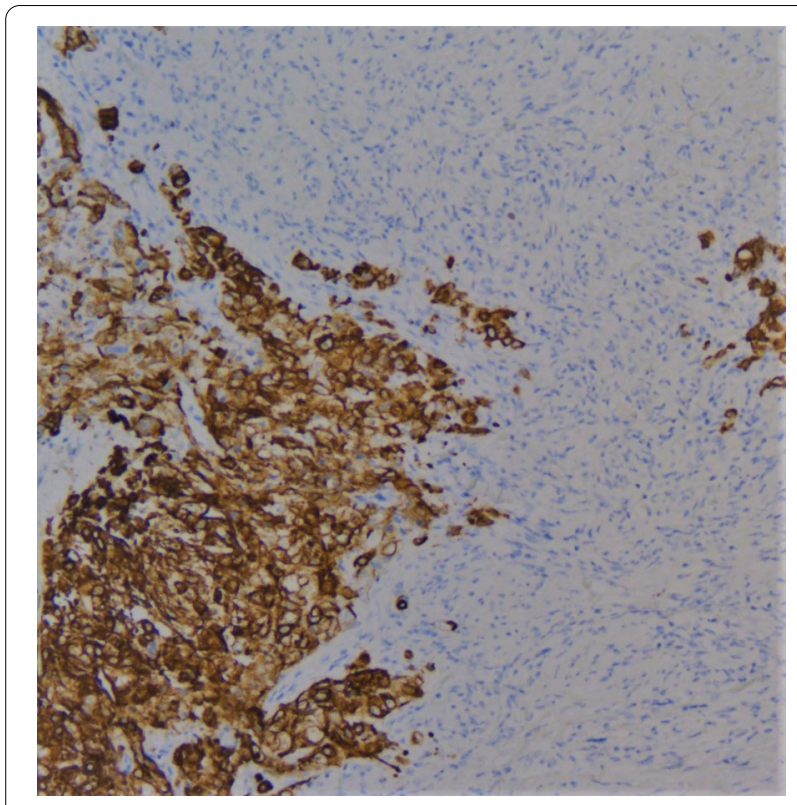

Fig. 3 Immunohistochemical (IHC) analysis of CK $5 / 6$ expression, 200x magnification

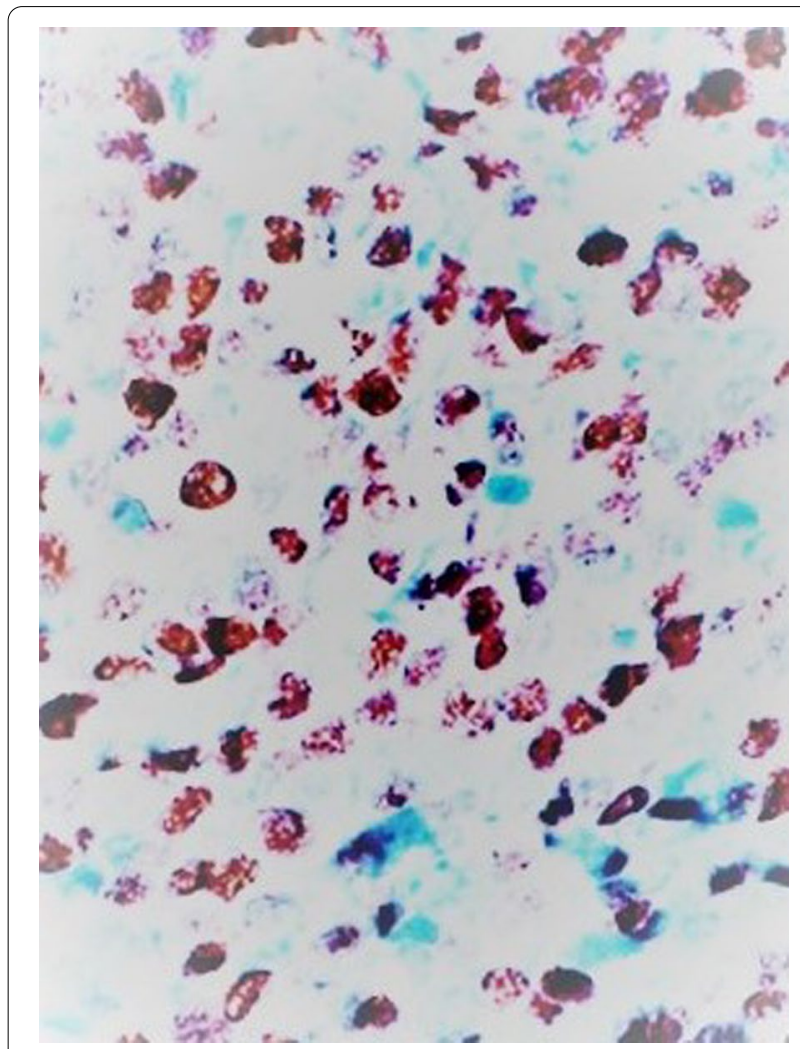

Fig. 4 Immunohistochemical $(\mathrm{IHC})$ analysis of myogenin expression, $400 \times$ magnification

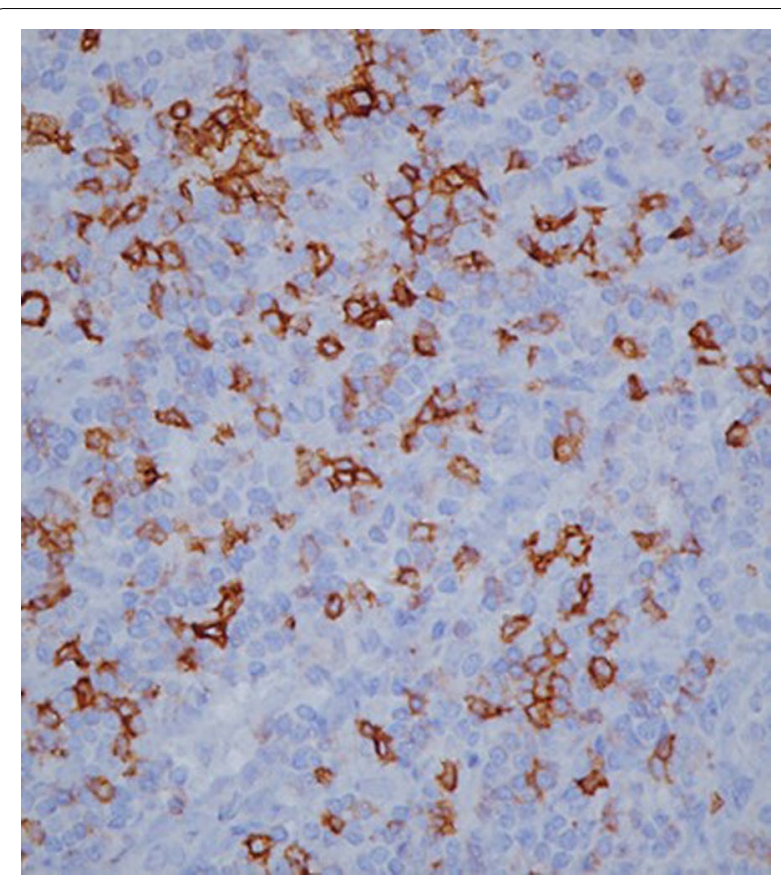

Fig. 5 Immunohistochemical (IHC) analysis of PD-L1 expression, $400 \times$ magnification

histopathological examination confirmed a pulmonary blastoma with malignant mesenchymal stroma showing slight foci of a rhabdomyosarcomatous differentiation pTNM stage IIIb (T4, N0, M0). The patient was qualified for six courses of adjuvant chemotherapy with vincristine, actinomycin-D, and cyclophosphamide. At the end of planned number of chemotherapy courses and 6 months after surgery the patient remained clinically and radiologically asymptomatic.

\section{Discussion}

Pulmonary blastoma $(\mathrm{PB})$ comprises a rare heterogeneous group of lung tumours typically containing immature epithelial and mesenchymal structures that imitate the embryonic lung tissue resembling fetal lung of gestational age $10-15$ weeks. PB accounts for $0.25-0.5 \%$ of all primary pulmonary malignancies. Based on the predominant tissue components, $\mathrm{PB}$ is divided into three subtypes:

- Well-differentiated fetal adenocarcinoma (WDFA), known as the monophasic pulmonary blastoma, consisting of epithelial component exclusively.

- Classic biphasic pulmonary blastoma (CBPB), comprising both epithelial and mesenchymal components, which is the most common subtype and currently considered as part of the spectrum of sarcomatoid carcinomas. 
- Pleuropulmonary blastoma (PPB), characterized by the presence of mesenchymal components only [2].

Historically, the term pulmonary blastoma (PB) had included both pure fetal adenocarcinomas, pleuropulmonary blastomas as well as the classic biphasic blastomas. However recent WHO re-classifications separated welldifferentiated fetal adenocarcinomas (WDFA) and pleuropulmonary blastomas (PPB) from the biphasic tumours (CBPB). Given the small number of cases and recent classification changes, interpreting the published clinical features and epidemiology of $\mathrm{PB}$ is challenging [3].

PB consists of an epithelial and mesenchymal stroma, where foci of chondrosarcoma, rabdomiosarcoma, osteosarcoma and yolk sac might also be found. Molecular studies indicate that epithelial and mesenchymal components may derive from a single precursor cell. Mutations in various genes (e.g., TP53, EGFR, CTNNB1) may be identified in some pulmonary blastomas [4]. Tumour specimens usually comprise areas of haemorrhage and necrosis, although translucent cytoplasm, tubular glandular cells and hyponuclear vacuoles might be demonstrated. The epithelial component of $\mathrm{PB}$ is composed mainly of tubules formed by non-ciliated glycogen-rich cells that mimic the pseudo-glandular stage of fetal lung development and typically does not express cytokeratins [5]. Different immunohistochemical staining methods in PB were studied by Larsen et al. The highest stain sensitivity was found with muscle actin (92\%), vimentin (90\%), neuron-specific enolase (NSE-83\%), $\alpha$-fetoprotein (AFP-82\%), carcinoembryonic antigen (CEA-77\%) and epithelial membrane antigen (EMA-71\%) [6].

The aetiology of $\mathrm{PB}$ remains unknown, however over $80 \%$ of cases are associated with a history of smoking. Abnormalities in laboratory tests are usually non-specific and rare. No specific tumour markers for PB have been found yet. Some reports presented elevated AFP and CEA serum levels [7].

The most frequent PB symptoms include cough, haemoptysis, chest pain, dyspnoea and fever of unknown origin, although $40 \%$ of cases remain asymptomatic and are revealed during incidental chest X-rays. In the vast majority of cases, the tumour is one-sided, with a higher prevalence in superior lobes. The mean tumour diameter at the time of primary diagnosis is $7-10 \mathrm{~cm}$. Typically radiological images show well-circumferenced mass displacing the mediastinum while CT-scans reveal dense and vesical elements with varying contrast uptake. Endobronchial growth is present in approximately $1 / 4$ of cases and even rarely pleura invasion is present. Differential diagnosis should include benign lesions such as pleural fibroma or hamartoma as well as other malignancies (primary lung cancers, metastases) [3, 8]. PB symptoms may occur at any age, but $80 \%$ of cases are diagnosed in adults, usually in the fourth decade of life and shows a strong female predominance, which is thought to be caused by the influence of estrogen through its receptors overactivated by $\beta$-catenin [9].

Diagnosis is made with transbronchial biopsy but obtaining the representative tissue sample is possible only in approximately $25 \%$ of cases because of the PB peripheral nature. Surgery is the preferred method of treatment as pulmonary blastoma typically is a welldemarcated peripheral mass. The range of surgery should be determined individually and depends on the tumour size, pleural invasion, lymph node metastasis and comorbidities. The average survival rate among operated patients is 33 months, as compared to 2 months' survival in non-operated patients. Limited lobectomies are associated with better survival rates than pneumonectomies, probably due to primary reduced tumour burden [10].

Larsen et al. reported a $16 \%$ response rate to chemotherapy in PB. So far, neither of the agents is more effective than another, although cisplatin is found in most treatment regimens, as it has been proved to improve prognosis in other germ cell tumours [6]. The selection of the most effective chemotherapeutics remains a major problem as the PB often presents biphasic structure with both epithelial and mesenchymal components, sensitive to different lines of treatment [11].

Overall PB prognosis is poor, the 2-year survival rate is $34 \%$, and the 5 -year survival rate is $16 \%$, respectively. Overall prognosis depends primarily on the tumour size and distant organs involvement. Biphasic type of tumour (CBPB), metastatic disease at the time of diagnosis, tumour size exceeding $5 \mathrm{~cm}$, early relapse (within 12 months after treatment) and lymph node involvement contribute to the unfavourable prognosis. At the time of diagnosis distant metastasis is present in $43 \%$ of patients and mainly concerns the brain, pleura, mediastinum, diaphragm and liver $[12,13]$.

Cancer in pregnancy is an increasingly common phenomenon faced by oncologist. This is a consequence of postponed motherhood until a later age and high rates of malignant tumors in the group of young women. Recent studies revealed the lack of knowledge among medical personnel and concerns about possible fetal damage caused by diagnostic radiology. Due to the possibilities of modern diagnostic equipment, also cancer radiological diagnosis in the first trimester of pregnancy is not contraindicated. According to Pereg et al. [14], performing radiological diagnostic procedures involving fetal exposure to ionizing radiation doses lower than 0.1 or even 0.2 Gy (10-20 cGy) does not increase the risk of congenital defects. The risk of birth defects decreases with increasing gestational age. 
Minimizing the effects caused by ionizing radiation by further reducing the dose and the residence time, increasing patient's distance from the radiation source as well as using a thicker shield and a less active radiation source allow the use of radiological imaging methods regardless of the stage of pregnancy $[14,15]$.

\section{Conclusions}

Pulmonary blastoma is an extremely rare malignant neoplasm characterized by poor prognosis. PB grows rapidly and patients' outcome relies on a rapid diagnosis what brings serious difficulties because of the rarity of the disease. Surgery remains the most common and effective treatment. Due to the extreme rarity, the literature contains only single mentions of $\mathrm{PB}$ in pregnancy, thus its impact on the course of pregnancy and the developing fetus remains unknown.

The presented case report is unique because it presents a very rare case of $\mathrm{PB}$ diagnosed during pregnancy, although it confirms that lung tumors should be included in the differential diagnosis of such patients, obviously after excluding other complications typical for the pregnancy.

The primary "take-away" lessons of this case report:

- Pregnancy is a state of physiological respiratory alkalosis and compensatory metabolic acidosis. The resulting acid-base disturbances are compensated by the kidneys. Low levels of $\mathrm{pCO} 2$ in the maternal arterial blood facilitate the uptake of oxygen by the placenta. It is therefore an adaptive change that ensures the optimal concentration of oxygen in the fetal circulation.

- Despite its very rare occurrence, the possibility of lung cancer in young pregnant women should not be forgotten.

- Haemoptysis in pregnant women, regardless of the stage of pregnancy, may be associated with the presence of a lung tumour.

- In the third trimester, the optimal management of pulmonary blastoma consists of delivery by caesarean section, stopping lactation and implementation of surgery treatment.

\section{Abbreviations}

PB: Pulmonary blastoma; WDFA: Well-differentiated fetal adenocarcinoma; CBPB: Classic biphasic pulmonary blastoma; PPB: Pleuropulmonary blastoma.

\section{Acknowledgements}

Not applicable.

\section{Authors' contributions}

MPB conceptualized the article and wrote the original draft. GP edited and reviewed the article. MB wrote the draft and prepared the review of the literature. $\mathrm{BO}$ prepared figures and reviewed the manuscript. EB collected data and reviewed the literature. ABK edited the manuscript and performed histological examination. All authors read and approved the final manuscript.

\section{Funding}

Not applicable.

\section{Availability of data and materials}

All data generated or analysed during this study are included in this published article.

\section{Declarations}

\section{Ethics approval and consent to participate}

Not applicable.

\section{Consent for publication}

Written informed consent was obtained from the patient for publication of this case report and any accompanying images.

\section{Competing interests}

The authors declare that they have no competing interests.

\section{Author details}

'Department of Gynaecologic Oncology and Obstetrics, Centre of Postgraduate Medical Education, 231 Czerniakowska, 00-416 Warsaw, Poland. ${ }^{2}$ Department of Cancer Prevention, Medical University of Warsaw, 81 Żwirki i Wigury, 02-091 Warsaw, Poland. ${ }^{3}$ Student Scientific Association, Medical University of Warsaw, 1 Oczki, 02-001 Warsaw, Poland.

Received: 18 June 2021 Accepted: 18 December 2021

Published online: 04 January 2022

\section{References}

1. Conrad KP. Mechanisms of renal vasodilation and hyperfiltration during pregnancy. J Soc Gynecol Investig. 2014;11:438-48. https://doi.org/10. 1016/j.jsgi.2004.05.002.

2. Wang XY, Zhang J, Chu XY, Liu Y, Li F, Wang BZ, et al. Diagnosis and multimodality treatment of adult pulmonary blastoma: analysis of 18 cases and review of the literature. Asian Pac J Trop Med. 2014;7:164-8. https:// doi.org/10.1016/S1995-7645(14)60015-8.

3. Smyth R, Fabre A, Dodd J, Bartosik W, Gallagher CG, McKone EF. Pulmonary blastoma: a case report and review of the literature. BMC Res Notes. 2014;13:294. https://doi.org/10.1186/1756-0500-7-294.

4. Zhao YY, Liu L, Zhou T, Zhou NN, Yang YP, Hou X, et al. A retrospective analysis of the clinicopathological and molecular characteristics of pulmonary blastoma. Onco Targets Ther. 2016;9:6915-20. https://doi.org/10. 2147/OTT.S117097.

5. Xiu Y, Jiang L, Liu W. Classic biphasic pulmonary blastoma with brain and axillary metastases: a case report with molecular analysis and review of the literature. Int J Clin Exp Pathol. 2015;8:983-8.

6. Larsen $\mathrm{H}$, Sorensen J. Pulmonary blastoma: a review with special emphasis on prognosis and treatment. Cancer Treat Rev. 1996;822:145-60. https://doi.org/10.1016/s0305-7372(96)90000-6.

7. Luo Z, Cao C, Xu N, Ying K. Classic biphasic pulmonary blastoma: a case report and review of the literature. J Int Med Res. 2020. https://doi.org/10. $1177 / 0300060520962394$

8. Lee HJ, Goo JM, Kim KW, Im JG, Kim JH. Pulmonary blastoma: radiologic findings in five patients. Clin Imaging. 2004;28:113-8. https://doi.org/10 1016/50899-7071(03)00240-7.

9. Vossler JD, Abdul-Ghani A. Pulmonary blastoma in an adult presenting with hemoptysis and hemothorax. Ann Thorac Surg. 2019;107:e345-7. https://doi.org/10.1016/j.athoracsur.2018.09.008. 
10. Van Loo S, Boeykens E, Stappaerts I, Rutsaert R. Classic biphasic pulmonary blastoma: a case report and review of the literature. Lung Cancer. 2011;73:127-32. https://doi.org/10.1016/j.lungcan.2011.03.018.

11. Kouvaris JR, Gogou PV, Papacharalampous XN, Kostara HJ, Balafouta MJ, Vlahos L. Solitary brain metastasis from classic biphasic pulmonary blastoma: a case report and review of the literature. Onkologie. 2006;29:56870. https://doi.org/10.1159/000096708.

12. Liman ST, Altinok T, Topcu S, Tastepe Al, Uzar A, Demircan S, et al. Survival of biphasic pulmonary blastoma. Resp Med. 2006;100:1174-9. https://doi. org/10.1016/j.rmed.2005.10.026.

13 Alahwal MS, Maniyar IH, Saleem F, Alshiekh M. Pulmonary blastoma: a rare primary lung malignancy. Case Rep Med. 2012. https://doi.org/10.1155/ 2012/471613.

14. Pereg D, Koren G, Lishner M. Cancer in pregnancy: gaps, challenges and solutions. Cancer Treat Rev. 2008;34:302-12.

15. Tirada N, Dreizin D, Khati NJ, Akin EA, Zeman RK. Imaging pregnant and lactating patients. Radiographics. 2015;35:1751-65. https://doi.org/10. 1148/rg.2015150031.

\section{Publisher's Note}

Springer Nature remains neutral with regard to jurisdictional claims in published maps and institutional affiliations.

- fast, convenient online submission

- thorough peer review by experienced researchers in your field

- rapid publication on acceptance

- support for research data, including large and complex data types

- gold Open Access which fosters wider collaboration and increased citations

- maximum visibility for your research: over $100 \mathrm{M}$ website views per year

At BMC, research is always in progress.

Learn more biomedcentral.com/submissions 